\title{
Views and approaches toward risks and benefits among doctors who become patients
}

\author{
Robert Klitzman * \\ Center for Bioethics, Columbia University, 1051 Riverside Drive, Unit 29, New York, NY 10032, USA
}

Received 17 May 2005; received in revised form 29 October 2005; accepted 11 November 2005

\begin{abstract}
Objective: To understand how views and approaches concerning risks and benefits may be affected by dynamic contexts and processes related to clinical roles and relationships.

Methods: We conducted two in-depth, semi-structured interviews with 50 doctors who became patients due to serious illnesses, concerning their experiences before and after diagnosis.

Results: As patients, these doctors gained critical new insights into the processes and contexts of communication about risks and benefits. Doctors and patients often varied in how they viewed and weighed risks and benefits (e.g., as "good' or "bad"). These data suggest a model in which patients undergo several dynamic processes: seeking statistics, accepting doctors' framing of statistics, being influenced by media hype, seeing statistics as relevant or not, over-valuing risks, weighing the importance of risks and benefits, interpreting statistics as good or bad, accepting or denying statistics and odds, and making treatment decisions. These processes are affected by external factors (e.g., doctors ordering tests, framing statistics, and often over- versus under-valuing risks), and internal factors (e.g., depression, denial of illness, optimism versus pessimism, magical thinking - that doctors are immune to disease - and rationalizations).

Conclusions: Doctors and patients are engaged in complex, dynamic processes that shape patients' approaches toward risks and benefits. Practice Implications: These data highlight the need for increased attention toward these issues in medical educational and care.

(C) 2005 Elsevier Ireland Ltd. All rights reserved.
\end{abstract}

Keywords: Risks; Benefits; Doctor-patient communication; Doctor-patient relationships; Qualitative research; Professional socialization; Medical education

\section{Introduction}

Patient comprehension of risk-benefit information takes place in the context of dynamic processes and relationships, but the effects and implications of these have been seriously understudied. Subjective factors that mold perceptions of statistics have been explored in several other fields, such as economics, but have been less examined in medicine. Kahneman received the 2002 Nobel Prize in Economics for suggesting that individuals commonly view probabilities subjectively, using biases and heuristics. For example, prospect theory suggests that "the response to losses is more extreme than the response to gains" [1]. In addition, the psychological "availability" and "retrievability" of exam-

\footnotetext{
* Tel.: +1 212740 7324; fax: +1 2127403508

E-mail address: rlk2@columbia.edu.
}

ples of a phenomenon can bias results such that individuals over-weigh rare, but traumatically-experienced outcomes [2]. Several psychological studies have probed such misperceptions of statistics-generally among college students and regarding hypothetical situations. For instance, research has shown that the interpretation of statistical information is affected by how it is framed, either positively or negatively [3], In addition, numerical estimates convey data more effectively than verbal descriptions alone [4].

In medicine, physicians and patients commonly confront significant uncertainties [5]. In these situations, biases and heuristics may distort patients' perceptions of risks [6]. Healthcare professionals can also present risks in varying ways. For example, research has shown that genetic counselors use a wide range of verbal descriptions [7]. Though different groups of medical personnel assign similar quantitative probabilities to qualitative description of odds 
[8], providers may differ significantly from patients in interpreting these words (e.g., "likely" versus "unlikely") [8]. Even when using numbers rather than verbal descriptions, patients may assess risks differently if presented rates rather than proportions [9]. The order in which risks and benefits are present can also influence responses [10].

Though some cognitive strategies used in medical decision-making have been explored [11,12], less attention has been paid to how dynamic factors and processes may shape the communication, interpretation, and understanding of risk/benefit information. Numerous interpersonal subjective factors may be involved in presentations and interpretations of risks and benefits, related to contexts and dynamic interactions of doctors and patients.

Doctors who become patients have received some attention, primarily focusing on single, anecdotal reports [13-16], and issues of self-doctoring [17,18]. Anecdotally, they have been forced to experience medical care from a different position, and thus come to see aspects of medicine from another perspective in ways that challenge their preconceived beliefs and expectations. But scant attention has been paid to how they view or alter their views of doctor-patient communication about risks and benefits. These doctor-patients are potentially unique and important in their ability to elucidate contrasts between the views and approaches of each group toward risks and benefits, having uniquely occupied both roles. They can elucidate issues that they were not previously aware of, but now recognize. Key questions thus arise, such as what perspectives and insights these doctor-patients arrive at, and how these relate to aspects of recent decision-making theory (e.g., by Kahneman and Tversky and others) $[1,2,12]$.

\section{Methods}

Pilot interviews were conducted, leading to the development and refinement of an interview guide. For the full study, subjects were recruited through emailed announcements (e.g., stating, "Are you or do you know a physician with a serious illness?"), websites, word of mouth, and ads in newsletters. As a result, the principal investigator (PI) was contacted by potential participants, including 48 doctors, 1 dentist, and 1 medical student who had become patients due to serious illnesses (referred to below as "doctors"). With each participant, two in-depth, semi-structured interviews of $2 \mathrm{~h}$ were held concerning experiences before and after diagnosis. Serious illness was self-defined, and then confirmed by the PI, a trained psychiatrist. Of these participants, 27 were HIV positive, and 23 had other medical problems (e.g., cancer, heart disease, and hepatitis). Ages ranged from 25 to 87, all were Caucasian, except for 1 Latino doctor, 40 were men, and 10 were women; and they were interviewed in several cities. The PI conducted all the interviews at participants' homes or offices, or in his office-whatever was more convenient for them. Partici- pants were asked about experiences as patients and as providers, and about other aspects of their lives.

The interviews were audiotaped, transcribed, and content-analyzed, informed by grounded theory [19]. Initial analyses were conducted during the period in which the interviews were being held. A research team, composed of the principal investigator and a research assistant, examined a subset of interviews to assess factors that shaped participants' experiences, identifying categories of recurrent themes and issues that were subsequently given codes. A senior consultant with expertise in qualitative research provided input at several stages of this coding process. The team assessed similarities and differences between participants, examining categories that emerged, ranges of variation within categories, and variables that may be involved. A coding manual was developed, and areas of disagreement were examined until consensus was reached. New themes that did not fit into this original coding framework were discussed, and modifications were made in the manual when deemed appropriate. In phase two of the analysis, the research team refined, merged, or subdivided thematic categories into secondary or sub-codes, when suggested by associations or overlap in the data. These codes and sub-codes were then used in analysis of all of the interviews. To ensure coding reliability, all interviews were analyzed by two coders.

The study was approved by the Institutional Review Board at Columbia University and the NY State Psychiatric Institute.

\section{Results}

These doctor-patients revealed a range of issues and problems as described below. Overall, their views and approaches toward risks and benefits appeared to be part of a process and were shaped by interpersonal, psychological, and temporal contexts. Heuristics and biases emerged in a variety of ways within these complicated interpersonal dynamics and processes that evolved over time.

Themes arose reflecting experiences as patients themselves, and as doctors treating other patients. They felt that their experiences as patients: were generally shared with lay patients, but, as noted below, at times, were more unique to doctor-patients. Invariably however, even themes that appeared in some ways specific to doctor-patients reflected larger underlying issues and concerns that lay patients shared as well. As illustrated on Fig. 1, the themes that emerged suggest a model, concerning factors involved in views and approaches toward risk and benefit information.

\subsection{Experiences as patients}

\subsubsection{Framing of statistics}

Many of these doctors became more aware of, and sensitive to, how statistics were framed within the context of 


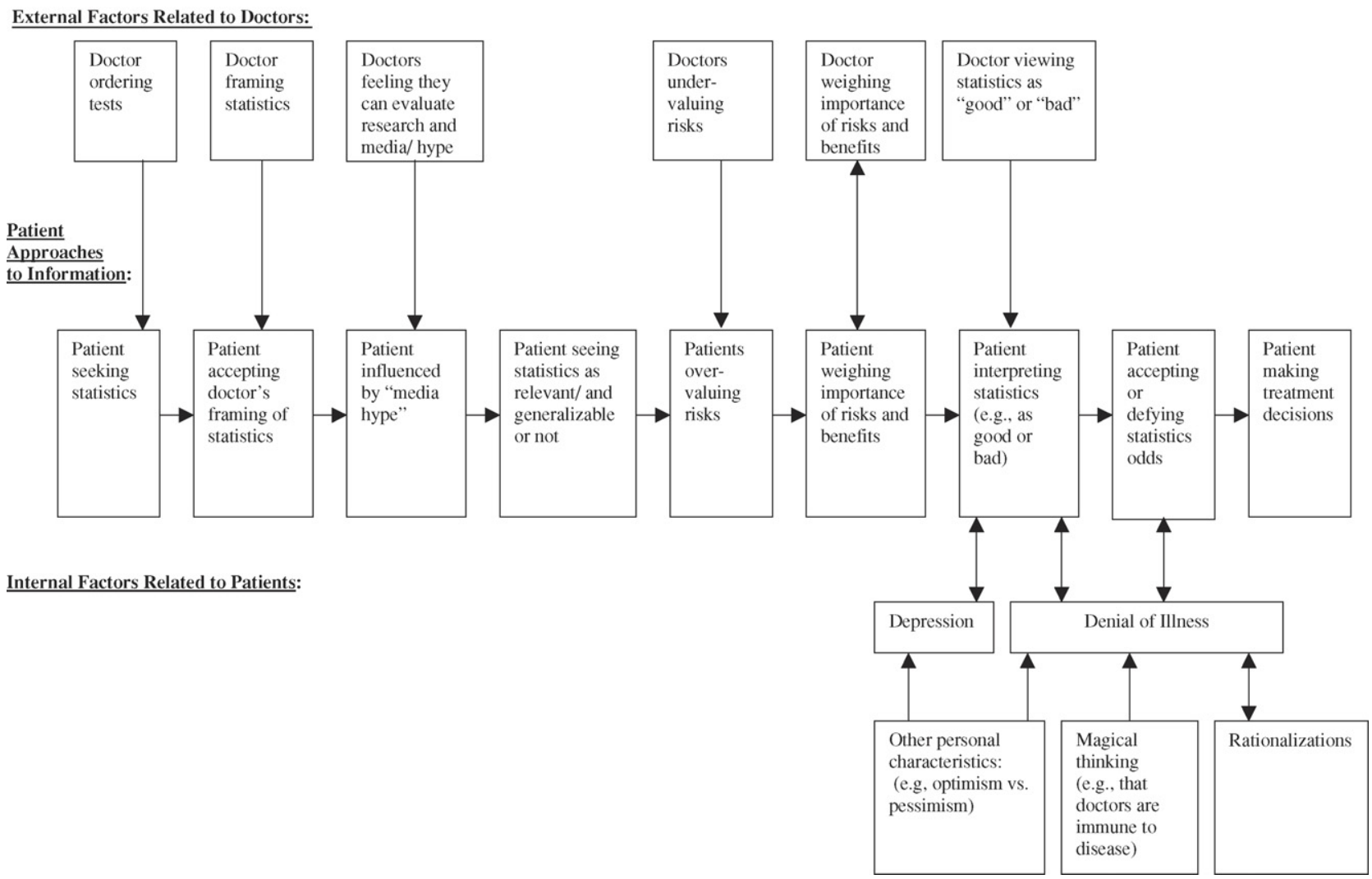

Fig. 1. Social, psychological, and temporal factors involved in views of risks and benefits.

doctor-patient relationships-not only what the statistics were, but also how they were presented. In particular, they felt providers' lack of empathy shaped presentations and thus perceptions of risk-benefit information. For example, one surgeon described how the night before his surgery, he was told by his physician that he had, "a 5\% chance of dying." He reported, "That night I couldn't sleep. If I had been told instead that I had a 95\% chance of surviving, I would have slept better." Surprise arose among these doctors concerning the need to frame data appropriately - i.e. as positively or negatively - and thus the extent to which emotions affected perceptions of risks and benefits.

These doctors became more aware, too, of the disparity between cognitive information, and emotional needs for support and reassurance. As one internist said, "I was surprised that I was as shocked and numb as I was when I found out I was HIV positive." Routinely, he had told patients who tested positive, "we don't know what the test means exactly, and there are good treatments-many patients do well for many years." But now having been diagnosed with HIV himself, none of these approaches felt helpful. Previously, he had viewed statistical and prognostic information as beneficial. He now felt that many physicians overvalued such information as almost "magically" therapeutic in and of itself, reflecting a sense of control over an illness. As patients, these doctors were surprised at how frequently they received "cold" presentations of data, yet they had difficulty conveying to their physicians desires for more emotional support.

\subsubsection{Interpreting statistics: over-versus under-valuing risks}

Tensions emerged as well concerning under- versus over-valuing risks. Generally, these doctor-patients felt that medical training increased their ability to access, understand, and interpret risk and benefit information. They felt they had "learned from clinical experience" - specifically that they had "gotten comfortable with risk," and "knew what the statistics meant" (i.e., that the existence of a danger did not necessarily mean that that untoward event would occur). They cited a common adage of medical training to temper biases in assessments of risks and overvaluation of the possibility of a negative outcome: "Common things happen commonly. Uncommon things happen uncommonly. You see the zebra diagnoses" medical conditions that are rare, but nonetheless described in detail in medical textbooks - "only once in a blue moon."

In contrast, these doctors generally thought that lay patients "tend to get hung up on risks of complications"that is feeling that a percentage likelihood of a complication happening means they will get it. Many of these doctorpatients believed they were less wary of risks of medications 
because of familiarity ("I'm not a pill wimp like some of my patients").

\subsubsection{Seeking and evaluating statistics}

Among these respondents, feelings arose that lay patients did not evaluate critically enough claims in the media and "media hype" about treatment and research findings. Some of these doctors felt that they, compared to patients, knew how to evaluate the research-not assuming that they would magically overcome the odds. (e.g., "I look at the literature, whereas other people may think they will be the exception.") Feelings emerged that, in fact, "the literature lies" - in part because only confirmatory results get published.

These doctor-patients varied in the extent to which they sought statistics related to their diseases. Though a few handled the intense anxiety of having a serious disease by "trying not to think about it," most sought to gather as much scientific data as possible, immediately checking on-line for information.

\subsubsection{Statistics as relevant or not}

Questions arose as well concerning the potential lack of "generalizability" of medical research: in estimating prognoses, research findings refer to group means, but not individual patients. Hence, at times these doctors felt that the applicability and generalizability of statistics to their own disease was open to interpretation. They felt that statistics helped physicians make decisions in general, but that for any patient, the relevant $N=1$. Thus, doctor-patients often did not 'identify' with these statistics, or feel these were relevant to them. Rationally, they knew that they were likely to follow the mean. But often, they came to realize more than before the potential limitations of medical knowledge, and that statistics may not be relevant.

Relatedly, patients' common questions of, "What would you do if you were me, doctor?" and, "What would you do if your mother was the patient?" were now seen as reflecting patients' efforts to apply population-based data to their own individual cases. Here, too, the interpretations of statistics had implications for treatment decisions, as well, in deciding how to act on risk information.

\subsubsection{Weighing risks}

These doctors now questioned, too, how to weigh risks. Specifically, a physician may recognize the existence of risk of a particular side effect, but weigh the importance of that risk differently than would a patient. For example, as patients themselves, many doctors perceived and appreciated their patients' side effects more, factoring in not just the therapeutic effects, but the psychological and social costs and suffering of side effects of treatments that, these doctors now felt, colleagues might underestimate. Often, these doctor-patients hadn't previously grasped precisely how distressing side effects could be. For example, these doctor-patients now became more aware of colleagues being overly "cavalier" about prescribing medications, minimizing the impact of particular adverse effects (e.g., weight gain) that are not medical diagnoses per se (i.e., with abnormal lab results that doctors can correct), and that their colleagues see as being volitional or relatively unimportant. For example, a psychiatrist who started on lithium for bipolar disorder, said that, for her, the hardest aspect of her treatment was weight gain (e.g., "That's the main problem: I'm 50 pounds overweight."). She lamented that her male colleagues in particular insufficiently appreciated both the importance of weight gain as a side effect, and its implications (e.g., difficulty finding a boyfriend).

\subsubsection{Interpreting risks as "Good" or "Bad"}

As patients, these interviewees faced difficult questions of how to interpret risks, especially those that were less than $50 \%$-that is, present, but not probable. Many sought subjective interpretations of quantitative data - conclusions that a particular risk was "good," "bad," or "great," (e.g., "do I have to worry or not?") - a more definitive status. As one internist said,

Did a $10 \%$ occlusion mean a low or high risk of arrhythmia? .... My big concern is: how bad is my heart disease? ... Radiologists say ... '60\% occlusion of your left marginal.' I have to ask specifically, 'How likely is a sudden arrhythmia?' ... You expect them to say ... 'Most of your circulation is good ... We assume it's dead or injured.'

Thus, despite their training, even these doctors found that, as patients, they were not always clear what "the numbers" in diagnostic tests meant in terms of clinical implications or prognoses. These doctor-patients wondered what constituted "good odds," (e.g., "There's no guide to thinking about how 'good' odds are not always good,") raising questions of how patients do and should make such determinations-e.g., how one should confront and respond to a $60 \%$ chance of survival $/ 40 \%$ chance of death.

\subsubsection{Accepting versus "defying" the odds}

As patients, some felt they might "defy" the odds, and be "lucky," and "exceptions" to the norm. Tendencies toward optimism and pessimism shaped assessments of risk. Fatalism arose in part based on seeing patients with similar diagnoses die (e.g., "It was a problem seeing a lot of sickness with patients. So I was pretty pessimistic. I assumed that in 5 years, I'd be facing severe disease and death"). These psychological tendencies could also reflect ingrained aspects of one's personality (e.g., "I've always been "fatalistic" or "I tend to be optimistic").

Pessimism was seen as having psychological advantages-not being let down. ("A pessimist is never disappointed, an optimist always is. So prepare for the worst and you're pleasantly surprised when it doesn't happen.") Despite efforts to remain "objective," and fight emotional biases in interpretations of data, pessimistic 
beliefs - at times irrational - could nonetheless seep in. Depression appeared to contribute to these interpretations. One doctor felt he had "bad luck," ("How could all this happen? Why me?") He had difficulty believing that he had not been somehow cursed, and he had to remind himself that chance events occur independently.

Conversely, feelings arose of being able to "defy the odds," that could verge on, or reinforce overconfidence and denial. Some stated that they felt they exercised "good denial" that was beneficial. But denial can foster procrastination and at times impeded preventative and other health behaviors; moreover, the difference between such "good" and "bad" denial was not always clear.

In considering risks, some indulged more explicitly in superstitious or magical thinking, particularly in beliefs that doctors are somehow immune from disease (e.g., "Doctor's don't get sick;" they "wear magic white coats." "You're either a doctor or a patient. You can't be both.") Such beliefs thus concerned not only prognoses, but also etiologies of disease.

As patients, many of these doctors realized that they often made decisions based not on "science," but on emotions-at times related to acceptance or denial of illness. For example, an internist with lymphoma received conflicting opinions from three different experts about treatment. In the end, he decided not to undergo a bone marrow transplant because he “just wasn't emotionally ready" to do so-i.e., he hadn't yet sufficiently accepted his disease and need for this procedure.

At times colleagues or loved ones challenged these doctor-patients' denial, and urged these ill doctors to pursue or adhere to treatment. Yet interviewees countered these attempts (e.g., "Leave me alone. I'm a doctor. I know what I'm doing.").

Nonetheless, worsening medical condition could increase pessimism. Optimism and denial of illness severity could remain unabated, however, "until the very end," altering only when evidence otherwise became irrefutable.

\subsection{Doctor-patients' experiences as physicians}

As doctors, these respondents described, too, several processes that appeared to shape, as external factors, their patients' views and approaches toward risk and benefit information.

\subsubsection{Ordering tests}

Frequently, though their awareness increased of the degree to which patients sought emotional comfort rather than cognitive understanding alone, these doctors nonetheless felt pressures to provide their own patients with quantitative data rather than emotional reassurance. Increasingly, they felt "procedure-oriented," in part because insurance companies reimbursed more for "high tech" procedures, than "low tech" physical exams, and most physicians had decreasing amounts of time with patients. Some felt that interns were particularly "procedure- oriented" because of having less clinical experience, and hence less confidence in clinical observations and interpretations. Tests provided ready answers, and doctors often felt more comfortable providing statistics, rather than more emotionally-charged interpretations (e.g., "good" or "bad").

\subsubsection{Doctors under-valuing risks}

These doctors felt that they at times underestimated and under-explained risks to their patients, especially iatrogenic risks, about which these doctors might feel guilty or uncomfortable. (e.g., "Medicine systematically underestimates the complications that we cause.") These doctors often sought to offer patients hope, and implicitly had a placebo effect. Yet professional training led some to feel comfortable dismissing small or negligible risks as "theoretical" (i.e., as ones that can be ignored in day-today practice). Still, with their patients, many of these doctors now, as a result of being patients themselves, felt less likely to "blow off mild symptoms" such as nausea or fatigue that might be "clinically insignificant" (i.e., not constituting a medical diagnosis per se) or "non-specific," but nonetheless distress the sufferer.

In interpretations of data to patients, to try to avoid ambiguity, some of these respondents now, as a result of their own illness, came to realize, too, the value and potency of visual images (e.g., radiographic studies of arterial occlusion), and some showed images to patients more than before.

Still, these doctors articulated professional barriers to appreciation of these issues by colleagues. Physician hubris can lead to conceptualizations of success and failure of treatment in self-affirming ways. An internist with cancer now criticized oncologists because of how they judged "good" outcomes and ignored side effects, over-reporting successes and downplaying failures, defining risks and benefits differently than patients might. He said:

What they call a success often is not, and is very ridiculous - they're giving someone three months more of a miserable life so that the patient can survive. But the medications have a lot of side effects that the oncologist never ... explained.

\section{Discussion and conclusion}

\subsection{Discussion}

Though much research on heuristics and biases has examined decision-making in isolation, and at a single point in time, these data suggest that approaches to risks and benefits are part of processes that transpire over time and are influenced by several critical, dynamic factors. These processes are affected, too, by external and internal variables. 
Risk and benefit information has been described as influencing a patient's affect, in turn shaping patient judgments about these risks and benefits [20,21]. Research in this area has generally been conducted among psychology students [22]. Yet the data presented here suggest that, at least among patients with serious illness, the process is more complex. Affect is also molded by personal experiences and characteristics, including psychological traits and states (e.g., underlying tendencies towards optimism or pessimism; and denial or acceptance of disease) that can in turn vary substantially over the course of an illness. Frequently in clinical practice, before a physician offers any new set of statistics, patients have already had symptoms. Difficult decisions arise of whether to proceed with additional treatments, given ongoing psychological, medical, and interpersonal processes (e.g., complex emotions of hope, optimism, fear, anxiety, depression, denial or acceptance of illness) that can interact with how statistics are understood. The data presented here thus suggest that statistical information about risks and benefits serves as a kind of Rorschach test that patients view and interpret in a variety of subjective ways, based on complex internal and external factors.

These results suggest, too, that physicians have complex a priori experiences, attitudes, and perspectives that can shape their approaches toward data in treating patients. These data parallel the finding that, indeed, physicians' practice style, e.g., not the prognosis of a case, affects the number of tests ordered for ICU patients [23].

Though heuristics and biases have been identified that physicians should avoid, these data illustrate how countervailing pressures arise that increase the use of these subjective factors. For example, prior studies have demonstrated that in presentations of data, numerical descriptors are more effective than verbal descriptors, and less subject to individual variation in interpretation, suggesting that genetic counselors and others avoid subjective, verbal expressions of statistics [3]. Yet even the doctor-patients here often used and relied on verbal presentations (e.g., about odds being "good" or "bad"), and desired such subjective interpretations of data, though these may be sub-optimal. Furthermore, even these verbal descriptors were not always easy to request from treating physicians.

The fact that, once becoming ill themselves, these doctorpatients were surprised by the significance of these issues is itself surprising, suggesting the extent to which professional socialization impedes awareness of such concerns. These doctors' use of statistics reflects an argot, a coded from of language with which they feel comfortable, and that reveals a set of attitudes and approaches toward illness and a process of probabilistic reasoning instilled in them through their training, both cognitively and emotionally. This language provides a sense of certitude, of answers, and of "covering themselves" against error due to misinterpretation, and reflects a desire to have control over the uncertainties and terrors of bad diagnoses. Doctors' desires to seek tests suggest that at times they may "hide behind the numbers," reflecting in part the reality that unknown factors may play key roles in treatment. Their use of statistics suggests, too, underlying attitudes about how uncertain future events may, in fact, be "known." Yet these doctors have been socialized to such an extent that they do not realize how much their discourse about statistics can be disconnected from the "reality" of patients' fears of diseases, and that ultimately, ambiguity still fills these phrases. Similarly, these doctors were at times surprised that statistics did not "shield" them emotionally, and that in several ways the field overrates medical information. They also realized they had low sensitivity to side effects that they had been trained to view as unimportant, and did not themselves experience.

This study has several potential limitations. The sample size may be small compared to certain studies, though for a qualitative study of this nature, it is large enough to provide insights into patterns of issues that emerge. These data are qualitative rather than quantitative, but as such can shed light on a range of crucial issues that future quantitative research can investigate in further detail with a larger sample. Though many of these doctors had HIV, given the added stigma involved, they convey several of these themes in bold relief. Of note, the issues that arose concerning their perspectives and approaches did not differ significantly from those of respondents with other kinds of medical disorders. Similar themes arose across the diseases faced. The themes that arose concerning risks and benefits also did not appear to differ based on participants' professional backgrounds. These data constitute perceptions of these doctors-I did not interview these respondents' own physicians as well to gain the latter's perspectives, which can be explored in future research.

\subsection{Conclusion}

To optimize clinical care, factors presented here must be taken into account in clinical practice, medical training, and future research on medical decision-making. Views and approaches toward risks and benefits can be profoundly shaped by many factors, including contexts of dynamic processes and relationships between doctors and patients.

Challenges emerge concerning how exactly medical education does and should address these issues-e.g., how trainees integrate observations of rare but serious complications with epidemiological data that such extreme complications are, in fact, uncommon. "Medical students' disease" has also been described, in which students often believe they are suffering from diseases about which they are learning, reflecting their anxiety about these disorders [24]. Yet, in the context of the present data, "Medical student's disease" can be viewed, in part, as a result of a bias due to overdiagnosing a condition because of anxiety about it, ignoring baseline rates of conditions. Various adages help trainees develop necessary intuition for overcoming biases and heuristics (e.g., "common things happen commonly" and 
"beware of "zebras"), Medical education also teaches the notion of "theoretical" risks that are low, but still exist. This minimization of potential but unlikely risks can help reduce biases, but can go too far, in that this process can distance providers from patients' experiences and perceptions in confronting risk and benefit information.

The experience of illness made these doctors realize how much patients hang on little turns of phrase. As patients, even these interviewees found it hard to provide feedback to their providers to present risks "less coldly." Patients face significant obstacles in attempting to improve physicians' approaches toward these issues. Hence, medical training needs to help doctors better understand what factors affect patients' experiences of illness and desires concerning risk/ benefit communication. Yet dilemmas remain of how best to incorporate into education the need for framing and empathetic presentations of risks.

Clearly, many of these issues in communication about risk information arise among lay patients as well. These doctor-patients occupy both roles, however, and can thus elucidate in bold relief many of these concerns and contrasts between doctors' and patients' perspectives. Their sensitivity to and perceptions of differences between themselves and lay patients reveal, too, their views of lay patients' approaches that may inform the care these doctors provide to others. Future research can probe similarities and differences with regard to lay patients.

These data can help, too, in future studies of heuristics and biases - on not only whether these operate, but also how exactly, and with what variations, given the complex contexts of interpersonal dynamics between doctors and patients, and emotional responses to disease. Patients' use of heuristics and biases may be informed, e.g., by physicians' tone, approach and assessments of risk and benefit data as "good" or "bad" (e.g., through anchoring of interpretations of data as described by Tversky and Kahneman) [1].

\subsection{Practice implications}

These data highlight the need to explain statistics carefully to patients, assess comprehension of probabilities, and understand the role of these complex processes and factors in doctor-patient relationships and communication. Attention must be paid to not only cognitive processing of statistics, but also underlying attitudes-e.g., how providers may see statistics as offering emotional solace and as being definitive and helpful in and of themselves. Providers should realize how patients may perceive these numbers differently than do doctors, and that statistics can raise more questions and anxieties than they resolve.

The fact that patients seek meaning in statistics (e.g., "do I have to worry or not?") places pressures on providers, and no doubt fuels the continued use of verbal expressions, despite the limitations of these. Such desire for certitude may also contribute to physicians ordering increasing numbers of tests and procedures in ways that may not be optimal, and that these providers need to be as aware of as possible.

In conveying statistical information, some of the doctors here suggested that visual images can be powerful ways of conveying risk and benefit data and thus can potentially be helpful in communicating risk information. This approach, and differences in perception between verbal or visual presentations of data has been explored somewhat [25], but needs to be further examined in future research.

I confirm that all patient/personal identifiers have been removed or disguised so the patient/person(s) described are not identifiable and cannot be identified through the details of the story.

\section{Acknowledgements}

The author would like to thank Renee C. Fox, Shaira Daya, and Mary DuVernay for their help with this project; and the National Institutes of Mental Health (through grant K08MH001420) and the Arthur Vining Davis Foundations for their support.

\section{References}

[1] Tversky A, Kahneman D. The framing of decisions and the psychology of choice. Science 1981;211:453-8.

[2] Kahneman D, Tversky A. Judgment under uncertainty: heuristics and biases. Science 1974;184:1124-31.

[3] Shiloh S, Sagi M. Effect of framing on the perception of genetic recurrence risks. Am J Med Genet 1989;33:130-5.

[4] Welkenhuysen M, Evers-Kiebooms G, d'Ydewalle G. The language of uncertainty in genetic risk communication: framing and verbal versus numerical information. Patient Educ Couns 2000;43:179-87.

[5] Fox R. Training for uncertainty. In: Merton RK, Reader GG, Kendall PL editors. The student physician. p. 207-41.

[6] Redelmeier D, Rozin P, Kanheman D. Understanding patients' decisions: cognitive and emotional perspectives. J Am Med 1993;270:72-6.

[7] Wertz D, Sorenson J, Heeren T. Clients' interpretation of risks provided in genetic counseling. Am J Hum Genet 1986;39:253-64.

[8] Kong A, Barnett G, Mosteller F, Youtz C. How medical professionals evaluate expressions of probability. N Engl J Med 1986;315:740-4.

[9] Grimes D, Snively G. Patients' understanding of medical risks: implications for genetic counseling. Obstet Gynecol 1999;93:910-4.

[10] Bergus GR, Levin IP, Elstein AS. Presenting risks and benefits to patients: the effect of information order on decision making. J Gen Intern Med 2002;17:612-7.

[11] Elstein AS. On the origins and development of evidence-based medicine and medical decision making. Inflamm Res 2004;53:s184-9.

[12] Elstein AS, Schwarz A. Evidence base of clinical diagnosis: clinical problem solving and diagnostic decision making: selective review of the cognitive literature. Br Med J 2002;324:729-32.

[13] Heymann J. Equal partners. Boston: Little Brown; 1995.

[14] Pinner M, Miller B, editors. When doctors are patients. New York: W.W. Norton; 1952.

[15] Mandell H, Spiro H, editors. When doctors get sick. New York: Plenum; 1987.

[16] Mullan F. Seasons of survival: reflections of a physician with cancer. N Engl J Med 1985;313:270-3.

[17] Fromme EK, Hebert RS, Carrese JA. Self-doctoring: a qualitative study of physicians with cancer. J Fam Pract 2004;53:299-306. 
[18] Fromme EK, Billings JA. Care of the dying doctor: on the other end of the stethoscope. J Am Med 2003;290:2048-55.

[19] Strauss A, Corbin J. Basics of qualitative research: techniques and procedures for developing grounded theory. Newbury Park: Sage Publications; 1990.

[20] Slovic P, Finucane ML, Peters E, MacGregor DG. Risk as analysis and risk as feelings: some thoughts about affect, reason, risk, and rationality. Risk Anal 2004;24:311-22.

[21] Finucane ML, Alhakami A, Slovic P, Johnson SM. The affect heuristic in judgments of risk and benefits. J Behav Decis Making 2000;13:117.
[22] Loewenstein GF, Weber EU, Hsee CK, Welch N. Risk as feelings. Psychol Bull 2001;127:267-86.

[23] Elstein AS, Christensen C, Cottrell J, Polson A, Ng M. Effects of prognosis, perceived benefit, and decision style on decision making and critical care on decision making in critical care. Crit Care Med 1999;27:58-65.

[24] Moss-Morris R, Petrie KJ. Redefining medical students' disease to reduce morbidity. Med Educ 2001;35:724-8.

[25] Moll JM. Doctor-patient communication in rheumatology: studies of visual and verbal perception using educational booklets and other graphic material. Ann Rheum Dis 1986;45:198-209. 